\title{
Data Enabled Predictive Energy Management of a PV-Battery Smart Home Nanogrid
}

\author{
Chao Sun, Fengchun Sun, and Scott J. Moura
}

\begin{abstract}
This paper proposes a data-enabled predictive energy management strategy for a smart home nanogrid (NG) that includes a photovoltaic system and second-life battery energy storage. The key novelty is utilizing data-based forecasts of future load demand, weather conditions, electricity price, and power plant $\mathrm{CO}_{2}$ emissions to improve the NG system efficiency. Specifically, a load demand forecast model is developed using an artificial neural network (ANN). The forecast model predicts load demand signals for a model predictive controller (MPC). Simulation results show that the data-enabled predictive energy management strategy achieves $96 \%-98 \%$ of the optimal NG performance derived via dynamic programming (DP). Its sensitivity to the control horizon length and load demand forecast accuracy are also investigated.
\end{abstract}

\section{INTRODUCTION}

The nanogrid and microgrid concepts have attracted significant interest for integrating distributed and renewable power generation into the smart grid [1]. Essentially speaking, a microgrid (MG) is a localized power system consisting of electric generation sources, loads, and energy storage connecting to the electric grid at a single point. Nanogrids (NGs) are small microgrids typically serving a single building, or a home in this paper's particular case. Economic viability and reliability of NGs depend critically on the energy management scheme, which determines flows of power between generation, loads, and storage. However, optimal energy management is complicated by uncertainty in environmental conditions, load demand, and battery aging. In this paper, we develop a predictive energy management scheme for a home with photovoltaics (PV) and second life battery energy storage, using data-based forecasting of environmental conditions, load demand, electricity prices, and grid emissions.

Rule-based energy management approaches have been widely studied for MG/NG applications. However, the design of such schemes depend on the designer's intimate knowledge of the MG/NG. Optimization-based energy management strategies are highly desirable. In particular, model predictive control (MPC) is appealing because it incorporates model-based predictions and explicitly enforces constraints [2]. The key challenge in MG/NG applications, however, is

C. Sun is a Ph.D. student of National Engineering Laboratory for Electric Vehicles, Beijing Institute of Technology, Beijing 100081, China. Now he is a visiting student researcher of Department of Mechanical Engineering, University of California, Berkeley, CA 94720, USA (chaosun.email@gmail.com).

F. Sun is with the National Engineering Laboratory for Electric Vehicles, Beijing Institute of Technology, Beijing 100081, China (sunfch@bit.edu.cn).

S. Moura is with the Department of Civil and Environmental Engineering, University of California, Berkeley, CA 94720, USA (smoura@ berkeley.edu). uncertainty in PV power generation and home load demand. One may forecast $\mathrm{PV}$ power via machine learning techniques [3] or irradiation and temperature forecasts in combination with photovoltaic system models [4]. Forecasting home load demand can be accomplished via machine learning [5], sensing individual plug loads [6], or physical models [7].

The main contribution of this paper is to systematically address load and solar power uncertainty by incorporating Internet-based weather data and forecasting methods into a model predictive control formulation of NG energy management. In particular, this paper adopts a data-driven approach to forecast future load demand using neural networks. PV power generation is predicted from photovoltaic models and existing data-based forecasts of environmental conditions, similar to [4].

The remainder of the paper is organized as follows. In Section II, the configuration and system model of the PVBattery smart home NG is presented. Section III develops and validates a data-enabled load forecast model. Section IV details the model predictive controller. Simulation results and sensitivity studies are illustrated in Section V, followed by key conclusions in Section VI.

\section{PV-GRID SMART HOME MICROGRID}

\section{A. Nanogrid Configuration}

In Fig. 1-(a) the smart home NG is composed of a PV array, a second-life ${ }^{1}$ lithium-ion battery pack, the home load demand, the utility grid, various power converters, data-based information and energy management algorithms. The battery is necessary to reconcile the imbalance between available PV power and load demand. The power flow topology is detailed in Fig. 1-(b). The PV panels and the battery are coupled to a DC bus to power DC loads. The DC bus is also connected to a DC/AC inverter to power AC loads and interact with the grid. Note that we assume energy will not be exported to the grid, since a robust regulation for selling electricity to the grid is absent yet. Compared with conventional homes, the PV-battery smart home NG provides additional degrees of freedom for electric power flow.

The controller's role is to manage power flow between these components to minimize objectives such as electricity cost or grid power plant emissions, subject to safe operating constraints. Specifically, a predictive scheme is applied that leverages Internet-based data to forecast home load demand and PV power. Next we detail sub-models for the NG components.

\footnotetext{
${ }^{1}$ the battery pack is reused from an automotive application, such as an electric vehicle
} 


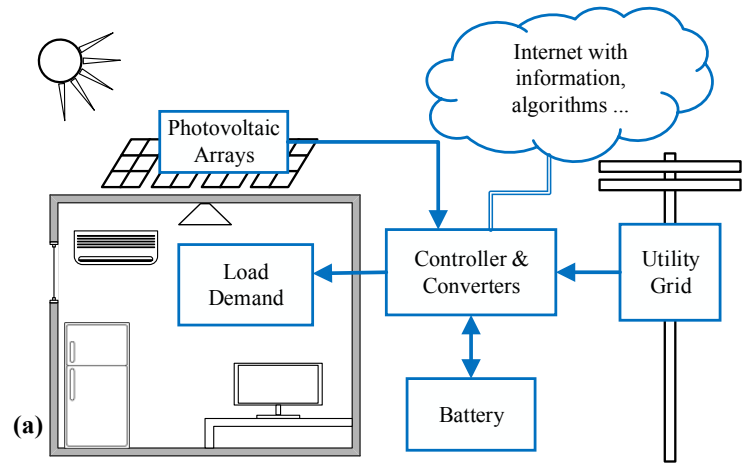

(b)

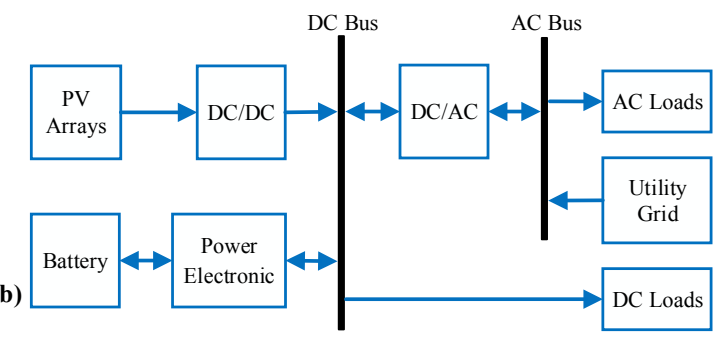

Fig. 1. A data-enabled PV-Grid smart home nanogrid: (a) the system configuration, where the Internet provides real-time data on weather conditions, load demand forecasts, electric cost rates, and other services for the controller; (b) the power topology.

\section{B. NG System Model}

1) Solar Irradiation: A Liu-Jordan model is adopted to determine the solar flux and PV panel temperature [8]. The solar irradiation includes the global horizontal irradiance $S_{g h}$, the direct beam irradiance $S_{d b}$, and the diffuse irradiance $S_{d i}$. The effective solar irradiance is given by

$$
\begin{array}{r}
S_{p v}=S_{d b}\left(\cos \theta_{s} \cos \beta_{p}+\sin \theta_{s} \sin \beta_{p} \cos \left(\alpha_{s}-\alpha_{p}\right)\right) \\
+S_{d i}\left(1+\cos \beta_{p}\right) / 2+S_{g h} \rho_{g}\left(1-\cos \beta_{p}\right) / 2,
\end{array}
$$

where $\theta_{s}$ and $\alpha_{s}$ are the zenith angle and azimuth angle of the sun, respectively; $\alpha_{p}$ and $\beta_{p}$ are the azimuth angle and altitude tilt angle of the PV panel, respectively; $\rho_{g}$ is the diffuse reflectance rate of the ground. The panel temperature is calculated by

$$
T_{p v}=S_{p v} e^{\left(a+b v_{w}\right)}+T_{a}
$$

where $T_{a}, v_{w}$ is ambient temperature and wind speed, and $a, b$ are empirical parameters.

2) Photovoltaic Array: The PV cell is modeled as an equivalent circuit [9]. This model consists of an ideal current source $I_{c s}$ in parallel with a diode and resistance $R_{p}$ all in series with resistor $R_{s}$. The diode models the semiconductor material, and $R_{s}$ models the resistance between the contactor and semiconductor material. The governing equations are given by

$$
\begin{aligned}
& V_{d}=V_{c e l l}+I_{p v} R_{s} \\
& I_{p v}=I_{c s}-I_{s}\left[e^{\left(\frac{q V_{d}}{A k T_{p v}}\right)}-1\right]-\frac{V_{d}}{R_{p}}
\end{aligned}
$$

$$
\begin{aligned}
& I_{s}=I_{s, r}\left(\frac{T_{p v}}{T_{r}}\right)^{3} e^{\frac{q E_{b g}}{A k}\left(\frac{1}{T_{r}}-\frac{1}{T_{p v}}\right)} \\
& I_{c s}=\left[I_{c s, r}+K_{I}\left(T_{p v}-T_{r}\right)\right] \frac{S_{p v}}{1000}
\end{aligned}
$$

where $V_{d}$ and $V_{\text {cell }}$ are the diode voltage and PV cell voltage, respectively; $I_{p v}$ is the $\mathrm{PV}$ cell output current, and $I_{s}$ is the cell saturation current; $q, A$ and $k$ are an electron charge, an ideal factor, and the Boltzmann's constant, respectively; $I_{s, r}$ is the cell's reverse saturation current at reference temperature $T_{r} ; E_{b g}$ is the band-gap energy of the semiconductor; $I_{c s, r}$ is the reference short-circuit current of the PV cell at $25^{\circ} \mathrm{C}$ and $1 \mathrm{~kW} / \mathrm{m}^{2} ; K_{I}$ is the cell's short-circuit current temperature coefficient. The cell model is scaled to an PV array by considering $n_{p v}$ cells in series [10], thus the array voltage and power are

$$
V_{p v}=n_{p v} V_{c e l l}, \quad P_{p v}=V_{p v} I_{p v}
$$

For brevity, we only summarize the PV model equations here from (3) to (7). Further details can be found in [10]. Note that a maximum power point tracking (MPPT) algorithm is required to improve PV efficiency. Details are omitted here for brevity.

3) Second-life Battery: Second-life batteries with $80 \%$ of the rated capacity are included in the NG. The battery pack is modeled as an equivalent circuit. The electrical power at the battery terminals is denoted as $P_{b a t t}$, and the battery state-of-charge is denoted by SOC. According to the power conservation law,

$$
P_{b a t t}=V_{o c} I_{b a t t}-I_{b a t t}^{2} R_{i n}, \quad \text { SOCC }=-\frac{I_{b a t t}}{Q},
$$

where $I_{b a t t}$ is the battery current; $V_{o c}, R_{i n}$ and $Q$ are the open circuit voltage, the internal resistance and the battery capacity, respectively. $P_{b a t t}>0$ corresponds to discharging, whereas $P_{b a t t}<0$ corresponds to charging. In practice, the parameters of the deployed second-life battery cells need to be re-identified through experiments, and additional efforts are required to eliminate the inconsistency between different cells [11].

4) Conservation of Power: The home power demand $P_{d e m}$ and grid utility power $P_{g r d}$ satisfy the power conservation law,

$$
P_{d e m}=P_{g r d}+\eta_{d d} \eta_{d a} P_{p v}+\eta_{d a} \eta_{b t} P_{b a t t}
$$

where $\eta_{d d}$ is the efficiency of the DC/DC converter; $\eta_{d a}$ is the efficiency of the DC/AC inverter (set as 1 when the power from PV or the battery is used to fulfill the DC load demands); $\eta_{b t}$ is the efficiency of the battery, $\eta_{b t}=\eta_{c h r g}$ when $P_{b a t t}<0, \eta_{b t}=\eta_{\text {dischrg }}$ when $P_{b a t t}>0$. $P_{\text {grd }} \geq 0$, meaning we cannot export power to the grid.

Equations (1)-(9) summarize the model used for the model predictive controller (see Section IV). Next we develop a data-based load demand forecasting algorithm. 


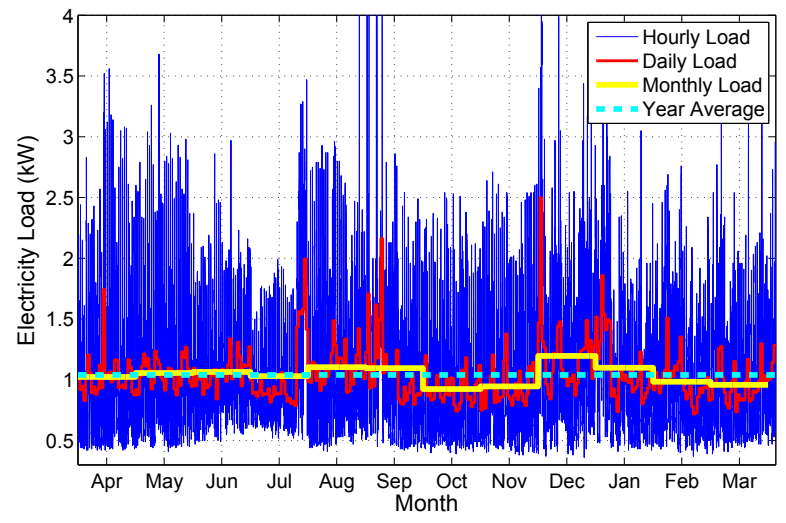

Fig. 2. Electricity consumption of a single family home in Los Angeles from 2013-04-01 to 2014-03-31.

\section{DATA ENABLED FORECAST}

\section{A. Load Data Analysis}

We analyze load data from a single family home in Los Angeles to investigate correlations between the load demand and the season, temperature, day of week, and time of day. The goal is to determine inputs to include in the data-driven model. The collected data corresponds to date range 2013-04-01 to 2014-03-31. Figure 2 plots the hourly, daily, monthly and yearly average electricity consumption. The hourly load demand varies between $0.5 \mathrm{~kW}$ to $4 \mathrm{~kW}$. The yearly average load is about $1 \mathrm{~kW}$. It is observed that the house consumed more energy in August and September (hottest months), and December, January (coldest months) relative to the other months. The correlation between the weekly average load and the weekly average temperature of this geographical area is also investigated. The result indicates that more energy is consumed when the weekly average temperature is higher than $21^{\circ} \mathrm{C}$ or lower than $14^{\circ} \mathrm{C}$. Since temperature correlates with different seasons, either input can be used for the forecasting model.

The load data is classified according to the day of week, shown in Fig. 3. We can see that from Monday to Thursday, the daily pattern of electricity consumption is similar. Peak loads consistently occur from 7:00 to 8:30 AM, and 6:00 to 10:00 PM. On Fridays, the pattern changes. There are two peak loads observed in the morning, which is clearly different from the Monday-Thursday pattern. During weekends, the electricity consumption pattern is more random. The peak loads on Saturday and Sunday usually last longer, and the daytime off-peak load is also higher compared with the weekdays, resulting with a higher average load during the weekend.

This analysis is used to determine the exogenous inputs of the forecast model (to be presented in Section III-B). Note that other information, such as the holidays or personal habits, can also be incorporated into the forecast model.

\section{B. Load Demand Forecast}

A radial basis function neural network (RBF-NN) forecast algorithm is utilized to forecast short-term future loads. RBF-

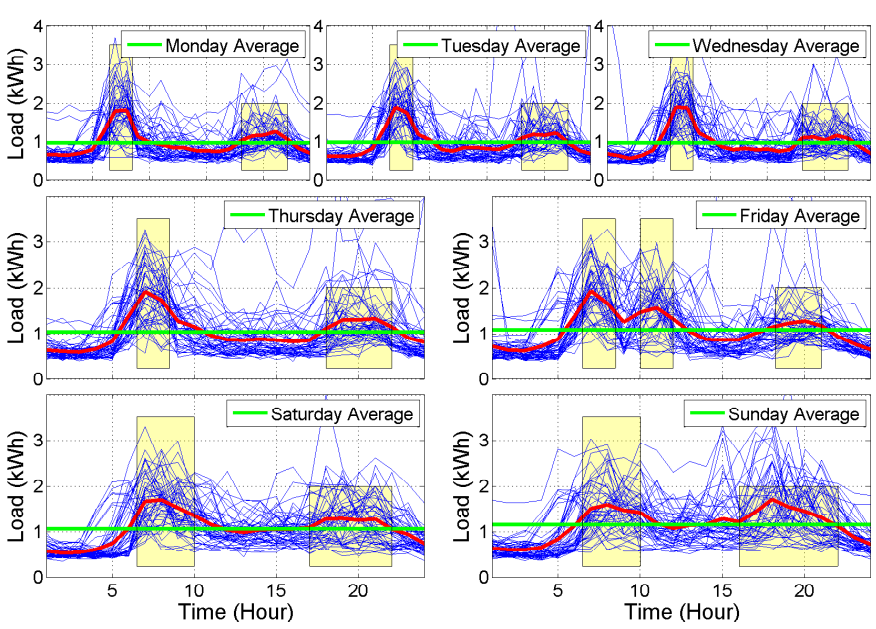

Fig. 3. Electric load from Monday to Sunday of the sampled LA data. Blue: load of particular week days; red: hourly average load across all weeks; green: daily average load over all weeks; yellow rectangle: peak load periods.

$\mathrm{NN}$ is selected because it captures the nonlinear input-output relations of home load demand and achieves reasonable forecast accuracy [12]. Please note that other forecast methods may be used. Generally, the RBF-NN model contains three layers: the input layer, the hidden layer, and the output layer. The hidden layer performs nonlinear transforms for feature extraction, and the output layer gives a linear combination of the output weights. The Gaussian function is used as the radial basis function in the hidden layer to activate the neurons [5], formulated as

$$
\begin{aligned}
a^{1} & =\exp \left(-\frac{\|n-c\|^{2}}{2 b^{2}}\right), \\
n & =W a^{0}+b
\end{aligned}
$$

where $a^{1}$ and $a^{0}$ are neural outputs of the current layer and prior layer, respectively; $n$ is accumulator output and $W$ is weight; $c$ is the neural net center and $b$ is the spread width. Both $c$ and $b$ can be fit using a gradient descent method.

Assume the input is $X$. According to Section III-A, the air temperature, day of week, and time of day are selected as exogenous inputs to the RBF-NN model, together with the endogenous input, the historical load. Thus, the input of the RBF-NN is designed as $X=\left[\begin{array}{llll}\hat{T}_{a} & D_{w} & T_{d} & L_{h}\end{array}\right]^{T}$, where $\hat{T}_{a}$ is the forecasted air temperature obtained via Internetbased weather services. $T_{a}$ is the true air temperature and used only during the training process. $D_{w}$ is the future day of week. $T_{d}$ is the future time of day. $L_{h}$ is the historical load. The output of the forecast algorithm $Y$ is the future $m$-dimensional load demand vector, notated as $\hat{P}_{d e m}$.

\section{Load Demand Forecast Validation}

The RBF-NN forecast model is validated in this section. The validation data is one-year real electricity consumption data (2013-04-01 to 2014-03-31) collected from two houses located in Los Angeles (LA) and Berkeley. The first half year data is used for neural network training, and the second 

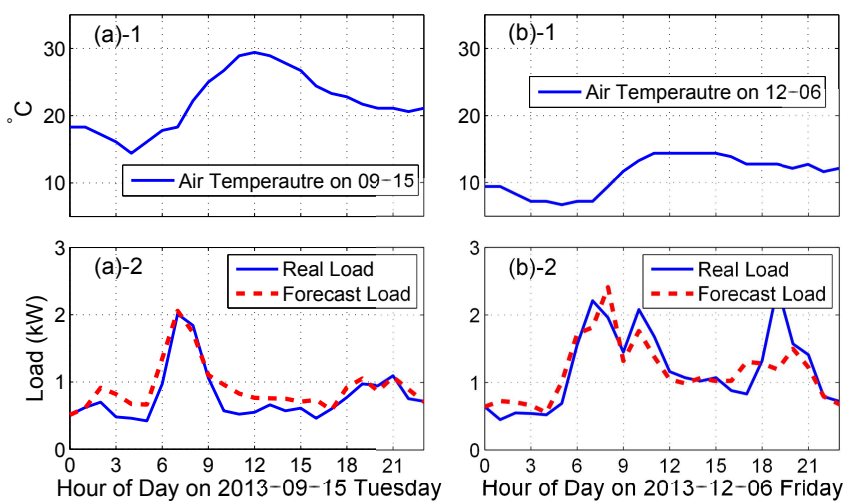

Fig. 4. Forecast examples and the corresponding air temperature of the LA load data: (a) on 2013-09-15 Tuesday; (b) on 2013-12-06 Friday. Note that the output vector length is 24-hour, meaning the forecast of the future 24-hour loads are completed in one process.
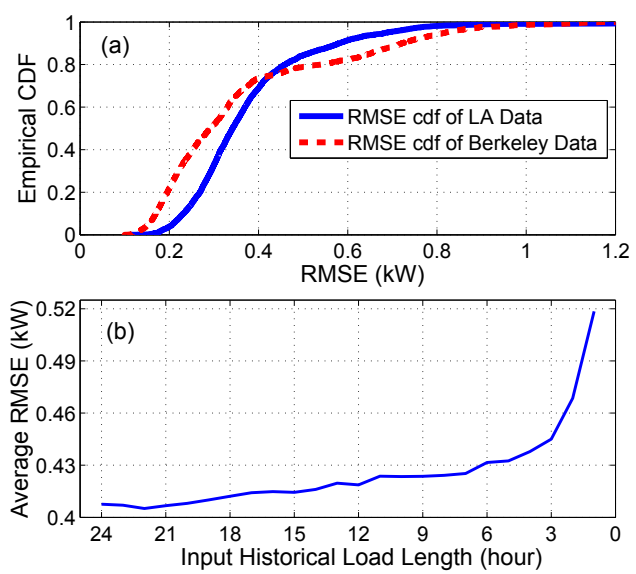

Fig. 5. (a): Empirical CDF for forecast RMSE of the LA and Berkeley load data; (b): Average RMSEs of the LA and Berkeley data with different historical load lengths used in the RBF-NN input (output is 24-hour long).

half year data is used for validation and comparison. The sampling period of the load data is one hour. The length of the historical load (in the input vector) and the length of the prediction horizon (output vector) are both initially set as 24 .

An empirical cumulative distribution function (CDF) of all the root mean square errors (RMSEs) of the forecast results are demonstrated in Fig. 5-(a). Note that 80\% of the RMSEs are below $0.45 \mathrm{~kW}$ and $0.55 \mathrm{~kW}$ in the LA and Berkeley data, respectively. The RBF-NN model is able to maintain the forecast error within an acceptable range. The accuracy validation of the forecast model is further quantified and verified in Section V.

Sensitivity to the input historical load length is also investigated. The average RMSE of the LA and Berkeley data with different historical load lengths is illustrated in Fig. 5-(b). As expected, longer historical load vectors produce better forecast accuracy. Interestingly, the marginal accuracy improvement decreases dramatically for historical load vectors greater than six hours. Conversely, the most recent five hours of load significantly impact forecasting accuracy.

\section{Weather, Cost, and Emission Forecasts}

Weather forecast services are now ubiquitous from the Internet. We assume that the solar irradiance and air temperature information is available via weather forecasting services (i.e. application programming interfaces (APIs)). The acquired irradiation and temperature information is injected into the solar irradiation model (see Section II-B.1) to estimate the PV solar flux and PV temperature, denoted as $\hat{S}_{p v}$ and $\hat{T}_{p v}$, respectively.

In addition, the electric cost and power plant carbon emissions are incorporated into the objective function of the controller (see Section IV). This information is also assumed to be available from the Internet. The observed electric rate and unit carbon emission are notated as $R_{e}$ and $C_{e}$, respectively.

\section{Model Predictive Control}

The proposed data-enabled predictive energy management strategy determines the optimal power flow, given forecasted load demand, weather conditions, and electricity cost obtained from the Internet. Given the system model (1)-(9), we require one control input to render a casual system, and select grid power $P_{\text {grd }}(t)$. Denoting $x(t)$ as the state variable, $u(t)$ as the control variable, $d(t)$ as the system disturbance, and $y(t)$ as the output, the system model is

$$
\begin{aligned}
\dot{x}(t) & =f(x(t), u(t), d(t)), \\
y(t) & =g(x(t), u(t), d(t)),
\end{aligned}
$$

with $x(t)=\operatorname{SOC}(t), u(t)=P_{g r d}(t), y(t)=P_{b a t t}(t)$. The disturbance $d(t)=\left[\hat{P}_{d e m}(t), \hat{S}_{p v}(t), \hat{T}_{p v}(t)\right]^{T}$, where $\hat{P}_{d e m}(t), \hat{S}_{p v}(t)$, and $\hat{T}_{p v}(t)$ are the forecasted load demand, solar irradiation and PV temperature, respectively. The electricity cost and carbon emission can be calculated by

$$
\begin{aligned}
& E_{r}(u, t)=R_{e}(t) \cdot u(t), \\
& E_{c}(u, t)=C_{e}(t) \cdot u(t),
\end{aligned}
$$

where $R_{e}(t)$ and $C_{e}(t)$ are time-varying electric rate and unit carbon emission, respectively. The objective function is

$$
E(u, t)=\lambda_{1} E_{r}(u, t)+\lambda_{2} E_{c}(u, t),
$$

where $\lambda_{1}$ and $\lambda_{2}$ are weighting parameters. For simplicity, we fix the prediction horizon length equal to the control horizon, namely $L_{p}$. Assume the time step is $\Delta t$. At time $k \Delta t$, the cost function $J_{k}$ is formulated as

$$
J_{k}=\int_{k \Delta t}^{\left(k+L_{p}\right) \Delta t} E(u, t)^{2} d t .
$$

Additionally, the following constraints must be satisfied:

$$
\begin{aligned}
& \mathrm{SOC}^{\mathrm{min}} \leq \mathrm{SOC} \leq \mathrm{SOC}^{\mathrm{max}}, I_{\text {batt }}^{\min } \leq I_{\text {batt }} \leq I_{\text {batt }}^{\max } \\
& P_{\text {batt }}^{\min } \leq P_{\text {batt }} \leq P_{\text {batt }}^{\max }, \quad P_{\text {grd }}^{\min } \leq P_{\text {grd }} \leq P_{\text {grd }}^{\max } .
\end{aligned}
$$

Special consideration is given to the battery terminal SOC constraint during each receding horizon of the MPC. Physically, the battery SOC can vary between SOC $^{\text {min }}$ and 


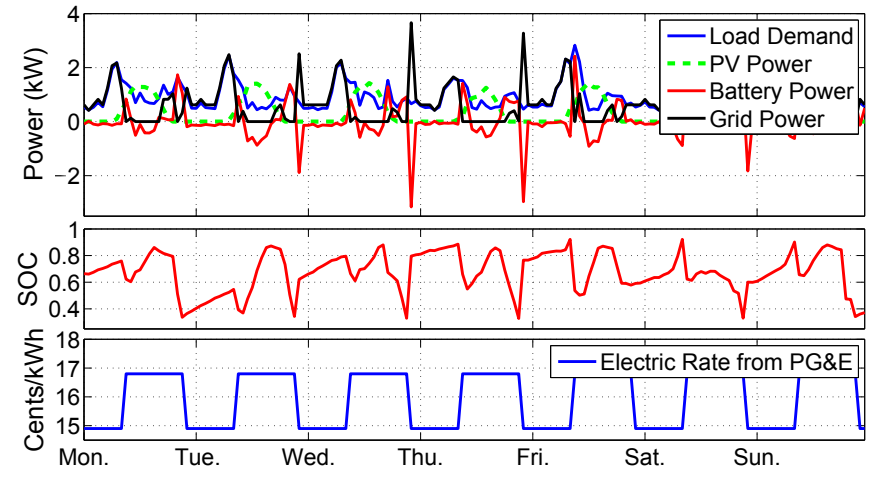

Fig. 6. Week-long control result of the data enabled predictive energy management on the LA house when $\lambda_{1}=1, \lambda_{2}=0$ in cost function (16), with load demand forecast by the RBF-NN.

SOC $^{\max }$ freely. However, in this paper we require the terminal SOC to be equal to a reference value, which is

$$
\operatorname{SOC}_{\left(k+L_{p}\right) \Delta t}=\overline{\mathrm{SOC}}
$$

where $\overline{\mathrm{SOC}}$ is a pre-defined reference for terminal SOC of each MPC horizon. In this case, the NG works under a charge-sustaining mode, to avoid overcharge or overdischarge situations. The control procedure of the proposed predictive energy management strategy follows the standard one of MPC [2].

Dynamic programming (DP) is employed in step two to solve the constrained nonlinear optimization problem at each time step. Alternative nonlinear formulations that admit special structure, e.g. convex programs or quadratic programs, can utilize corresponding solvers [13]. DP is used here for its generality and provable optimality.

\section{Simulation And Discussion}

\section{A. Data-enabled Predictive Energy Management}

The PV panel parameters are adopted from a commercial product: Renogy Monocrystalline 250D. The battery pack is from a Toyota Prius hybrid electric vehicle, with 168 series-arranged C-LiFePO 4 cells. Each cell's nominal charge capacity is $6.5 \mathrm{Ah}$, and we assume the second-life pack has already degraded to $80 \%$ of its original energy capacity. We consider $6 \mathrm{PV}$ panels in series and 5 battery packs in parallel. Note that the energy management strategy focuses on slower dynamics of the NG, thus the control time step is selected as 1 hour. Faster dynamics are governed by a lower level controller in practice.

1) Supervisory Control: The control and prediction horizon length are 24 hours. The electricity load data is collected data from single family homes in LA and Berkeley. The load demand during each control horizon is predicted by the RBF-NN forecast model. The weather condition, solar flux, electric rate and carbon emission data are obtained from the National Climatic Data Center, PG\&E and CAISO, respectively. These information streams are assumed known to the controller via the Internet.

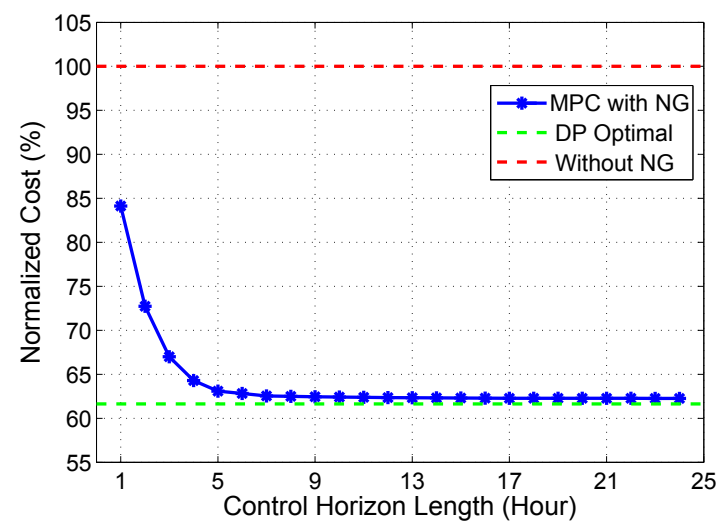

Fig. 7. Normalized energy management performance when increasing the control horizon length from 1 to 24 , where CM stands for the proposed dataenabled predictive energy management strategy. The cost without PV/battery is normalized as $100 \%$ to indicate the worst case performance. Dp optimal is the best-case solution solved by DP when full knowledge is known a priori.

First, we consider $\lambda_{1}=1, \lambda_{2}=0$ in the cost function (16) to investigate the optimal NG behavior with respect to electric cost only. A week-long energy management result is shown in Fig. 6. The PV power is intermittent, and drops to zero during the night. During the day, the solar energy is directly used to power the house. Extra energy is stored into the battery for future use. When solar energy is insufficient to satisfy load demand, the battery or grid provides support. The bottom figure shows a two-tiered cost structure, including high-cost "on-peak" rates and lower-cost "off-peak" rates. To reduce the electricity cost, the controller avoids on-peak grid power as much as possible, as demonstrated in Fig. 6. Consequently the battery generally charges during off-peak periods, and discharges during on-peak periods.

A similar simulation result is observed where the objective is to minimize the carbon emissions $E_{c}$, i.e. $\lambda_{1}=0, \lambda_{2}=1$. The controller avoids using grid power during higher carbonemission periods.

2) Horizon Length Determination: Next we examine control horizon length. The energy management performance for control horizons of $1 \mathrm{hr}$. to $25 \mathrm{hrs}$. are shown in Fig. 7, normalized to the electric cost without PV and battery (i.e. without NG). For a $1 \mathrm{hr}$. horizon, the MPC is short-sighted and normalized cost is about $85 \%$. As the control length increases, the performance converges towards the lower bound calculated via dynamic programming (DP) with global time horizon and perfect forecasts (DP optimal). When the control length is 7 hours, the cost is $2 \%$ greater than the DP result (approximately 64\%). Consequently, performance nearly equal to having perfect forecasts (i.e. the DP result) can be achieved with a 7 hour horizon, with negligible marginal improvements for longer control horizons.

3) Energy Manager Assessment: Ten weeks are randomly selected from the LA and Berkeley data sets for a comprehensive assessment of the controller. Results are listed in Table I. Symbols $C, P$ and $\sigma$ indicate the cost or carbon in USD or kg, percentage, and the standard deviation, 
TABLE I

PERFormance COMPARISON WITH RESPECT TO COST AND CARBON

\begin{tabular}{ccccccc}
\hline \hline Type & $C_{r t}$ & $P_{r t}$ & $\sigma_{r t}$ & $C_{c b}$ & $P_{c b}$ & $\sigma_{c b}$ \\
\hline Without NG & 27.32 & $100 \%$ & - & 8.03 & $100 \%$ & - \\
DP with NG & 16.97 & $62.1 \%$ & $+/-0.3 \%$ & 4.93 & $61.4 \%$ & $+/-0.7 \%$ \\
CM with NG & 17.54 & $64.2 \%$ & $+/-0.8 \%$ & 5.07 & $63.1 \%$ & $+/-1.2 \%$
\end{tabular}

( $r t$ indicated for the electric rate, and $c b$ means the carbon emission.)

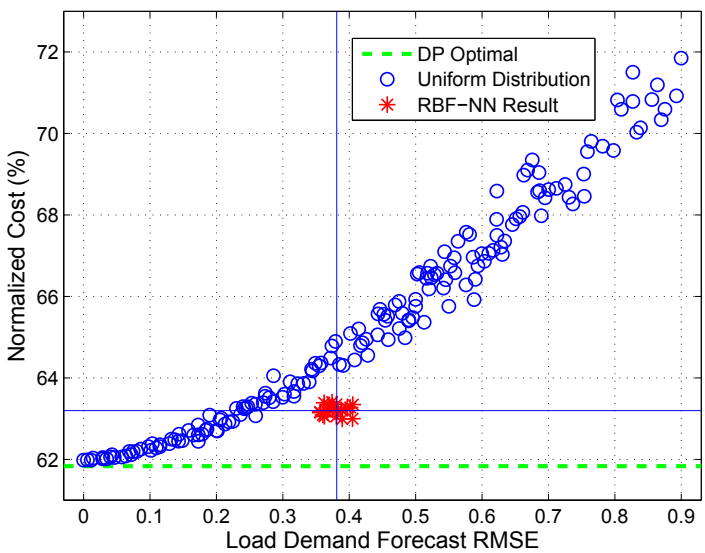

Fig. 8. Normalized energy management performance of the artificially formulated load demand with uniformly distributed RMSEs from 0 to 1 , compared with the RBF-NN energy management results.

respectively. We can see that both the electricity cost and carbon emission can be reduced by over $35 \%$ compared to homes without a NG system. Moreover, the data-enabled predictive energy management is only $2 \%$ worse than the DP optimal benchmark. This suggests moderately accurate forecasts of load demand are sufficient for excellent controller

4) Forecast Error Sensitivity Study: Next we investigate load demand forecasting error on energy management performance. To conduct this sensitivity study, we append uniformly distributed random errors to the real load demand data. The RMSE of this artificially formulated load is increased from 0 to $1(\mathrm{~kW})$. We implant the contaminated load demand forecasts into the MPC.

Over 200 tests with uniformly distributed errors are conducted, compared with 20 RBF-NN tests, shown in Fig. 8. When the RMSE is below $0.3 \mathrm{~kW}$, the energy manager performs closely to the DP optimal solution, with normalized costs are between $62 \%$ and $64 \%$ compared with no NG scenario. These results demonstrate that normalized cost increases linearly as the forecast RMSE increases.

Additionally, we note the RMSE of the RBF-NN forecaster is near $0.38 \mathrm{~kW}$. The normalized cost is $63.4 \%$, which equals the performance of contaminated forecasts with just $0.25 \mathrm{~kW}$ RMSE. This result is surprisingly good, which is only $1.5-2 \%$ higher than the DP optimal solution. After comparison, we found that the forecast RMSE produced by the RBF-NN has a tighter distribution (i.e. smaller variance) compared to uniformly distributed errors. This indicates that the RBF-NN forecast model captures the nonlinear performance.

characteristics of the load data and provides a reasonable disturbance estimate for MPC, relative to the performance achieved with perfect forecasts.

\section{CONCLUSIONS}

This paper presents a data enabled predictive energy management strategy for a PV-battery smart home nanogrid. A model predictive controller (MPC) is formulated to solve the energy management problem. A RBF-NN model is utilized to forecast home load demand, which is used as a disturbance prediction in the MPC controller. Future weather conditions and other information streams are acquired from the Internet integrated into the energy management system. Numerical experiments demonstrate that the proposed predictive energy management system achieves $96 \%-98 \%$ optimality of the deterministic DP benchmark with respect to electric cost and carbon emission. In addition, the controller's sensitivity to the control horizon length and load demand forecast accuracy are investigated to determine the fundamental tradeoffs. Future work involves energy storage from plug-in electric vehicles, and demonstration of the proposed energy management strategy on an experimental smart home at the University of California, Davis [11].

\section{REFERENCES}

[1] N. Hatziargyriou, H. Asano, R. Iravani, and C. Marnay, "Microgrids," IEEE Power and Energy Magazine, vol. 5, no. 4, pp. 78-94, 2007.

[2] E. F. Camacho and C. B. Alba, Model Predictive Control. Springer, 2013.

[3] A. Chaouachi, R. M. Kamel, R. Andoulsi, and K. Nagasaka, "Multiobjective intelligent energy management for a microgrid," IEEE Transactions on Industrial Electronics, vol. 60, no. 4, pp. 1688-1699, 2013

[4] E. Perez, H. Beltran, N. Aparicio, and P. Rodriguez, "Predictive power control for PV plants with energy storage," IEEE Transactions on Sustainable Energy, vol. 4, no. 2, pp. 482-490, 2013.

[5] H. S. Hippert, C. E. Pedreira, and R. C. Souza, "Neural networks for short-term load forecasting: A review and evaluation," IEEE Transactions on Power Systems, vol. 16, no. 1, pp. 44-55, 2001.

[6] Y. Suhara, T. Nakabe, G. Mine, and H. Nishi, "Distributed demand side management system for home energy management," in 36th Annual Conference on IEEE Industrial Electronics Society. IEEE, 2010, pp. 2430-2435.

[7] D. Zhang, L. G. Papageorgiou, N. J. Samsatli, and N. Shah, "Optimal scheduling of smart homes energy consumption with microgrid," in The First International Conference on Smart Grids, Green Communications and IT Energy-aware Technologies, 2011, pp. 70-75.

[8] B. Liu and R. Jordan, "Daily insolation on surfaces tilted towards equator," ASHRAE J. (United States), vol. 10, 1961.

[9] G. Vachtsevanos and K. Kalaitzakis, "A hybrid photovoltaic simulator for utility interactive studies," IEEE Transactions on Energy Conversion, no. 2, pp. 227-231, 1987.

[10] S. J. Moura and Y. A. Chang, "Lyapunov-based switched extremum seeking for photovoltaic power maximization," Control Engineering Practice, vol. 21, no. 7, pp. 971-980, 2013.

[11] S. J. Tong, A. Same, M. A. Kootstra, and J. W. Park, "Off-grid photovoltaic vehicle charge using second life lithium batteries: An experimental and numerical investigation," Applied Energy, vol. 104, pp. 740-750, 2013.

[12] N. Amjady, "Short-term hourly load forecasting using time-series modeling with peak load estimation capability," IEEE Transactions on Power Systems, vol. 16, no. 3, pp. 498-505, 2001.

[13] S. Boyd and L. Vandenberghe, Convex optimization. Cambridge university press, 2009. 